Research Article

\title{
Spermatogenesis of Zaprionus indianus and Zaprionus sepsoides (Diptera, Drosophilidae): Cytochemical, structural and ultrastructural characterization
}

\author{
Letícia do Nascimento Andrade de Almeida Rego, Rosana Silistino-Souza, \\ Maria Tercília Vilela de Azeredo-Oliveira and Lilian Madi-Ravazzi \\ Departamento de Biologia, Instituto de Biociências, Letras e Ciências Exatas, \\ Universidade Estadual Paulista “Julio Mesquita Filho”, São José do Rio Preto, SP, Brazil.
}

\begin{abstract}
Zaprionus indianus is a drosophilid native to the Afrotropical region that has colonized South America and exhibits a wide geographical distribution. In contrast, $Z$. sepsoides is restricted to certain African regions. The two species differ in the size of their testes, which are larger in $Z$. indianus than in $Z$. sepsoides. To better understand the biology and the degree of differentiation of these species, the current study evaluated spermatogenesis in males of different ages by conventional staining techniques and ultrastructural analysis. Spermatogenesis and the ultrastructure of spermatozoa were similar in the two species, and the diploid number was confirmed to be $2 n=12$. A greater number of spermatozoa were observed in young $Z$. indianus (1-3 days old) compared to $Z$. sepsoides males, which showed a higher frequency of cells at the early stages of spermatogenesis. The head of the sperm was strongly marked by silver staining, lacto-acetic orcein and the Feulgen reaction; the P.A.S. reaction revealed glycogen granules in the testes of both species. Both species presented similar arrangement of microtubules $(9+9+2)$, two mitochondrial derivatives of different size and 64 spermatozoa per bundle. Such similarity within the genus Zaprionus with other species of Drosophila, indicates that these structures are conserved in the family Drosophilidae. The differences observed the number and frequency of sperm cells in the early stages of spermatogenesis, between the young males of $Z$. indianus and $Z$. sepsoides, are features that may interfere with reproductive success and be related to the invasive potential of $Z$. indianus.
\end{abstract}

Keywords: Zaprionus indianus, Zaprionus sepsoides, spermatogenesis, cytochemistry, ultrastructure, meiosis.

Received: July 5, 2012; Accepted: October 15, 2012.

\section{Introduction}

The genus Zaprionus is divided into two biogeographically separate subgenera: the subgenus Anaprionus Okada, with approximately 10 species in the Eastern region, and the Zaprionus subgenera, with approximately 50 species in the Afrotropical region (Okada and Carson, 1983; Yassin et al., 2008). Phylogenetically, Zaprionus is very close to the genus Drosophila (Yassin et al., 2007, 2008, 2010; Yassin and David, 2010).

The species Zaprionus indianus (Gupta, 1970) originated in Africa was first identified in Brazil in 1999 and is considered an invasive species that has infested fig plantations (Vilela, 1999; Tidon et al., 2003). After 1999, Z. indianus has been detected in different regions of Brazil (Toni et al., 2001, Castro and Valente, 2001; Santos et al., 2003; Kato et al., 2004; Mattos-Machado et al., 2005, Da-

Send correspondence to Lilian Madi-Ravazzi. Laboratório de Genética, Ecologia e Evolução de Drosophila, Departamento de Biologia, Instituto de Biociências, Letras e Ciências Exatas, Universidade Estadual Paulista "Julio Mesquita Filho", São José do Rio Preto, SP, Brazil. E-mail: lilian@ @ibilce.unesp.br. vid et al., 2006), Uruguay (Goñi et al., 2001) and, in 2005, Florida (USA) (Van der Linde et al., 2006) and Argentina (Soto et al., 2006).

Studies examining the invasive potential of $Z$. indianus, included its life cycle (Amoudi et al., 1991; Stein et al., 2003), larval competition (Amoudi et al., 1993a) and aspects of fitness (Amoudi et al., 1993b; Setta and Carareto, 2005). Allozyme studies have indicated that plasticity in the distribution of the allelic frequency at the Est-3 locus may have also contributed to the success of this species in spreading across the Americas (Galego and Carareto, 2007, 2010). However, few studies have examined the biology and reproduction of $Z$. indianus (Araripe et al., 2004) or other Zaprionus species (Yassin et al., 2007; Yassin and David, 2010; Yassin et al., 2010).

Of the 49 species of the genus Zaprionus, only three have become invasive: $Z$. indianus, $Z$. tuberculatus and $Z$. ghesquierei (Chassagnard and Kraaijeveld, 1991). Z. indianus is the most widespread species of the genus and the ecologically most diverse of the Afrotropical drosophilid fauna (Yassin et al., 2007, 2010; Yassin and David, 2010). The general habits of this species may be a major 
factor in its successful occupancy of four continents (Lachaise and Tsacas 1983, Schmitz et al., 2007). However, other factors, including aspects of its reproduction, adaptation and ecology, may also be involved in the invasion process.

Male characteristics that influence the competitive ability to mate and fertilization success can also interfere with the fitness of the species. For example, sperm size is a male trait that is predicted to be under strong sexual selection (Hosken et al., 2003, Snook, 2005; Scharer et al., 2008), even though the selective advantage of different sizes of sperm remains largely unclear (García-González and Simmons, 2007; Amitin and Pitnick, 2007). The number of sperm and their developmental stages are also factors that can contribute to the reproductive potential of the species.

The present study aims to expand on previous studies on the biology of $Z$. indianus and the characteristics that possibly influence its adaptation to various environments, as reflected by its successful invasion of different continents. More specifically, this paper analyzes spermatogenesis and sperm ultrastructure of a geographical strain of Z. indianus and of Z. sepsoides (Duda, 1939). Z. sepsoides is restricted to certain regions of East Africa and differs from $Z$. indianus in testes size and sperm. Furthermore, it is not considered an invasive species.

\section{Material and Methods}

\section{Origin of insects}

A strain of $Z$. indianus from Ubatuba, SP, Brazil, and a strain of $Z$. sepsoides originating from the Congo region of Africa were used in the present work. These strains were maintained at $\pm 25^{\circ} \mathrm{C}$ in standard culture medium: banana and yeast (Saccharomyces cerevisiae).

\section{Measurements of testes}

Pairs of testes were dissected from 12 eight-day-old adult males of each species for obtaining linear measurements. For such measurements, the spiral testes from $Z$. indianus were first uncoiled.

\section{Preparation of slides and cytochemical techniques}

In this analysis, the testes were removed from males of different ages (1-8 days of life) from both species. The testes were stained using lacto-acetic orcein following De Vaio et al. (1985) and impregnated with silver nitrate following Howell and Black (1980), with modifications. After the usual preparation of the slides, the procedure for the Feulgen reaction was followed according to Mello and Vidal (1980), with modifications, for both species.

The structural and ultrastructural analyses were performed according to Cotta-Pereira et al. (1976), with modifications. The testes of adult $Z$. indianus and $Z$. sepsoides males were removed at different developmental stages (1,
3,5 and 8 days old) and fixed in a solution of $4 \%$ paraformaldehyde and $2.5 \%$ glutaraldehyde in $0.2 \mathrm{M}$ phosphate buffer, $\mathrm{pH}$ 7.4. After initial fixation for $4 \mathrm{~h}$ at $4{ }^{\circ} \mathrm{C}$, the testes were washed three times in $0.1 \mathrm{M}$ phosphate buffer, $\mathrm{pH}$ 7.4 , for $15 \mathrm{~min}$ each. Next, the material was post-fixed in a $1 \%$ osmium tetroxide solution for $2 \mathrm{~h}$ in a dark, sealed bottle in the refrigerator. After this procedure, the material was washed three times in distilled water for $5 \mathrm{~min}$ each and then dehydrated in a series of increasingly concentrated acetone solutions $(30 \%, 50 \%, 70 \%$ and $90 \%$ acetone), with 15 min per solution, followed by dehydration in $95 \%$ and $100 \%$ acetone three times for $15 \mathrm{~min}$ each. After dehydration, the material was embedded in a 1:1 mixture of resin (Araldite) and 100\% acetone for infiltration overnight at room temperature. The next day, the material was removed from the resin-acetone solution, placed in resin and placed in a $37^{\circ} \mathrm{C}$ oven for $2 \mathrm{~h}$. The testes were then removed from the bottles containing the pure resin, and the embedding process was initiated in the mold, which was placed in a $60{ }^{\circ} \mathrm{C}$ oven for $72 \mathrm{~h}$ for polymerization.

Semi-thin, $0.5 \mu \mathrm{m}$ sections of the testes were stained with $1 \%$ toluidine blue and analyzed using a light microscope. Ultra-thin, $70 \mathrm{~nm}$ sections were contrasted with uranyl acetate and lead citrate and analyzed by transmission electron microscopy.

For periodic acid-Schiff (P.A.S.) staining, the slides were first prepared for structural analysis for the two species, as follows (Mello and Vidal, 1980, with modifications). A solution of $0.5 \%$ periodic acid was applied to the semi-thin, $0.5 \mu \mathrm{m}$ sections, which were then placed in a $60{ }^{\circ} \mathrm{C}$ oven for $14 \mathrm{~min}$. Next, the sections were washed in distilled running water for $5 \mathrm{~min}$. A Schiff reagent was added to slides, which were protected from light and were placed in a $60{ }^{\circ} \mathrm{C}$ oven for $45 \mathrm{~min}$. Following the washing of each sample in 3 distilled water baths for 5 min each; the slides were air-dried and mounted with nail polish.

The material prepared by means of conventional cytochemical and staining techniques was analyzed using a light microscope (Olympus BX40) and Axiovision LE digital imaging software, Version 4.8 for Windows. The material prepared using histological techniques was analyzed and photographed using a JEOL 1011 transmission electron microscope operated at $80 \mathrm{kV}$.

\section{Results}

\section{Testes morphology of Z. indianus and Z. sepsoides males}

The testes of $Z$. indianus and $Z$. sepsoides are yellow in color (Figures $1 \mathrm{~A}$ and $1 \mathrm{C}$, respectively). In Z. indianus, the testes consist of two coiled tubes of approximately $5 \mathrm{~mm}$ in length each (Figure 1A), whereas in Z. sepsoides, the testes are two kidney-shaped tubes of approximately $2 \mathrm{~mm}$ in length each (Figure 1C). 


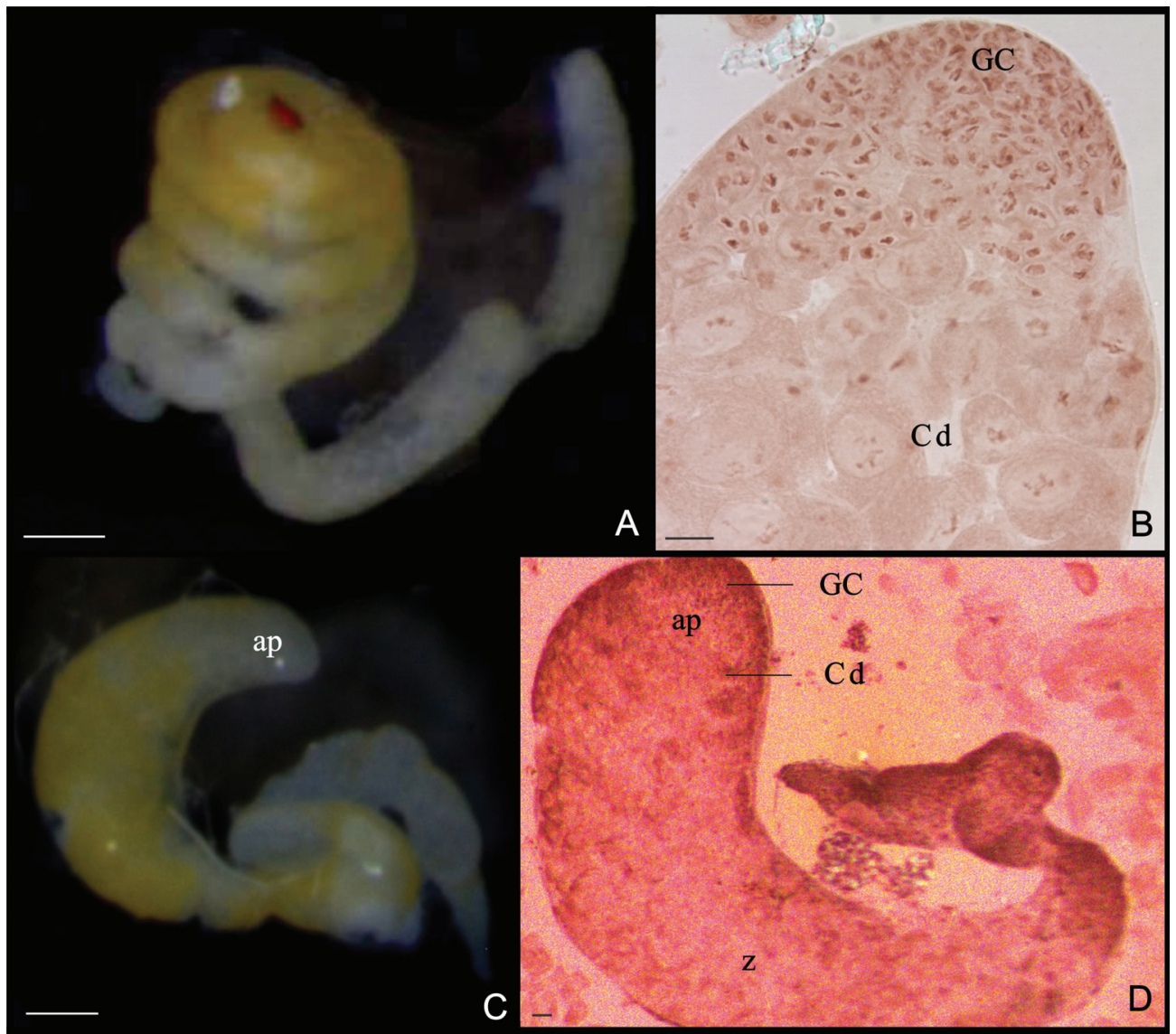

Figure 1 - Testes and cells of Zaprionus indianus (A-B) and Zaprionus sepsoides (C-D). A and C. Testes seen under a stereo microscope. B and $\mathbf{D}$. Squash staining with lacto-aceto orcein showing the germinal center (GC) in the apex of the testes (ap), followed by cells in division (Cd) and spermatozoids $(\mathrm{z})$ present in the central region of the testis. Scale bars: A and C $(0.5 \mathrm{~mm})$; B and D $(10 \mu \mathrm{m})$.

\section{Lacto-acetic orcein staining and Feulgen reaction}

In the apical part of the testes, a large number of dividing cells was observed. During prophase I, the nuclei of the primary spermatocytes in the diplotene and diakinesis stages exhibited chromosomes that were arranged in a circular ring and were associated with each other. The sex chromosomes were separate from this ring, as indicated by arrows in Figures 2A and 2J.

The metaphase stage of meiosis I revealed six pairs of bivalent chromosomes $(2 n=12)$ in the nucleus in a circular arrangement (Figures $2 \mathrm{~B}-\mathrm{C}, 2 \mathrm{E}-\mathrm{F}$ and $2 \mathrm{~K}$ and $2 \mathrm{~L}$ ).

At the end of telophase I, the formation of two nuclei was observed (Figures 2D, 2G and 2M). During spermiogenesis, the spermatids become elongated (Figures $2 \mathrm{H}$ and $2 \mathrm{~N}$ ) and organized into long bundles in $Z$. indianus (Figure 2I) and short bundles in $Z$. sepsoides (Figure 2O). In $Z$. sepsoides, these bundles stained strongly and revealed a concentration of genetic material at their tips, corresponding to the nuclei of spermatozoids during individualization (Figure 2O). This strong staining indicated the presence of aldehyde groups, based on the color produced by the reactive Schiff stain.
Mainly primary spermatocytes were observed in the apical part of the testes of $Z$. indianus and $Z$. sepsoides, whereas in other parts of the testes, secondary spermatocytes, spermatids and spermatozoa were noted (Figures 1B and 1D).

\section{Impregnation by AgNOR}

Cells in various stages of spermatogenesis were observed by impregnation with AgNOR. For both species, spermatocytes in the early stages of prophase I were observed, with nucleolar corpuscles scattered throughout the cell nucleus (Figures 2P and 2S). Spermatids at later stages of elongation showed no impregnation with AgNOR along their entire length (Figures 2Q and 2T), whereas spermatozoa arranged into long bundles and strongly stained with AgNOR in their extremities were frequently observed in $Z$. indianus (Figure 2R). In contrast, in Z. sepsoides, sperm bundles did not exhibit this staining pattern (Figure 2U).

\section{Structural and ultrastructural analysis of the testes and spermatogenesis of $Z$. indianus and $Z$. sepsoides}

The testes of $Z$. sepsoides and $Z$. indianus were analyzed at different stages of development $(1,3,5$ and 8 days 
old). In Z. sepsoides, the dividing cells (spermatocytes) and elongating cells (spermatids) were more easily detected in young males (1-3 days old), whereas in older ones spermatozoids were noted more frequently. In contrast, in $Z$. indianus, a greater abundance of sperm bundles was observed in young males (1-3 days old), with only few cells in the early stages of spermatogenesis.

In the testes of both species, diverse and unique cellular structures were found. The germinal center was the location of a considerable number of germline stem cells, which were identified at the apex of the testis in both Zaprionus species. The testicular content could be divided into two parts: germline stem cells at the apex of the testis and the remainder of the testes filled with cells corresponding to other stages of spermatogenesis. The testicle is coated by a layer of cells and more internally by an envelope (Figures 1B, 1D, 3A, 3B, 4A, 4C and 5A). Strongly stained granules were also observed, particularly in the testes of $Z$. sepsoides. These granules may contain glycogen as in- ferred from the results of the P.A.S. reaction on these testes (Figure 3B).

The successive stages of spermatogenesis were not visible in the testes of $Z$. indianus, perhaps due to their spiral morphology and size, which hampered their proper preparation as ultra-thin sections. Alternatively, the peak of the initial stages may have occurred earlier in development, for instance in the pupal stage. In Z. sepsoides, some stages of spermatogenesis were observed, with dividing cells occupying the entire apical portion of the testis together with the presence of many granules. Additionally, in regions such as the testicular apex, many circular and elongated spermatids were observed, and in other regions, bundles of spermatozoa were distributed in all directions (Figures $3 \mathrm{~A}-\mathrm{B})$.

The differentiation of the spermatids in both species within the cysts is synchronous (Figures 4A-B and 5A-B). Another observation at ultrastructural level was the consis-
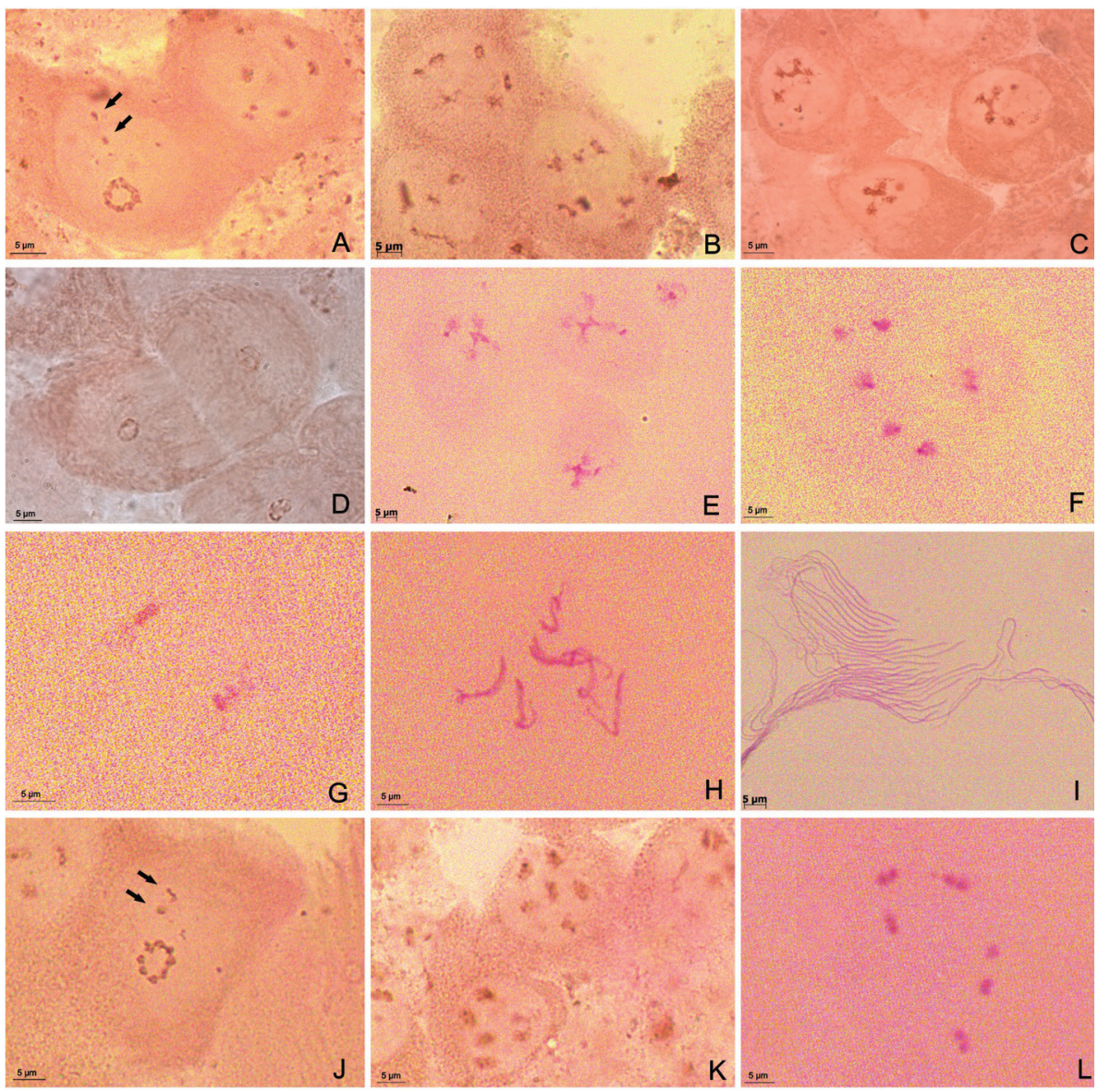

Figure 2 - Histological and cytochemical staining results. Zaprionus indianus cells stained using lacto-aceto orcein (A-D) and subjected to the Feulgen reaction (E-I). Zaprionus sepsoides cells stained using lacto-aceto orcein (J, K, M) and subjected to the Feulgen reaction (L, N, O). Testicular cells of Zaprionus indianus (P-R) and Zaprionus sepsoides (S-U) after silver impregnation. A. Prophase I (diplotene / diakinesis phase), showing the chromosomes arranged in a circular ring and the sex chromosomes (arrows) as separate from this ring. B, C, E, F. Metaphase I of meiosis showing the six pairs of bivalent chromosomes. D, G. Telophase I showing the genetic material in the center of each nucleus. H. Elongated spermatids I. Spermatozoids organized into long bundles. J. Prophase I (diplotene / diakinesis phase), showing the chromosomes arranged in a circular ring and the sex chromosomes (arrows) as separate entities from this ring. K, L. Metaphase I of meiosis showing the six pairs of bivalent chromosomes. Scale bars: $5 \mu$ m. 


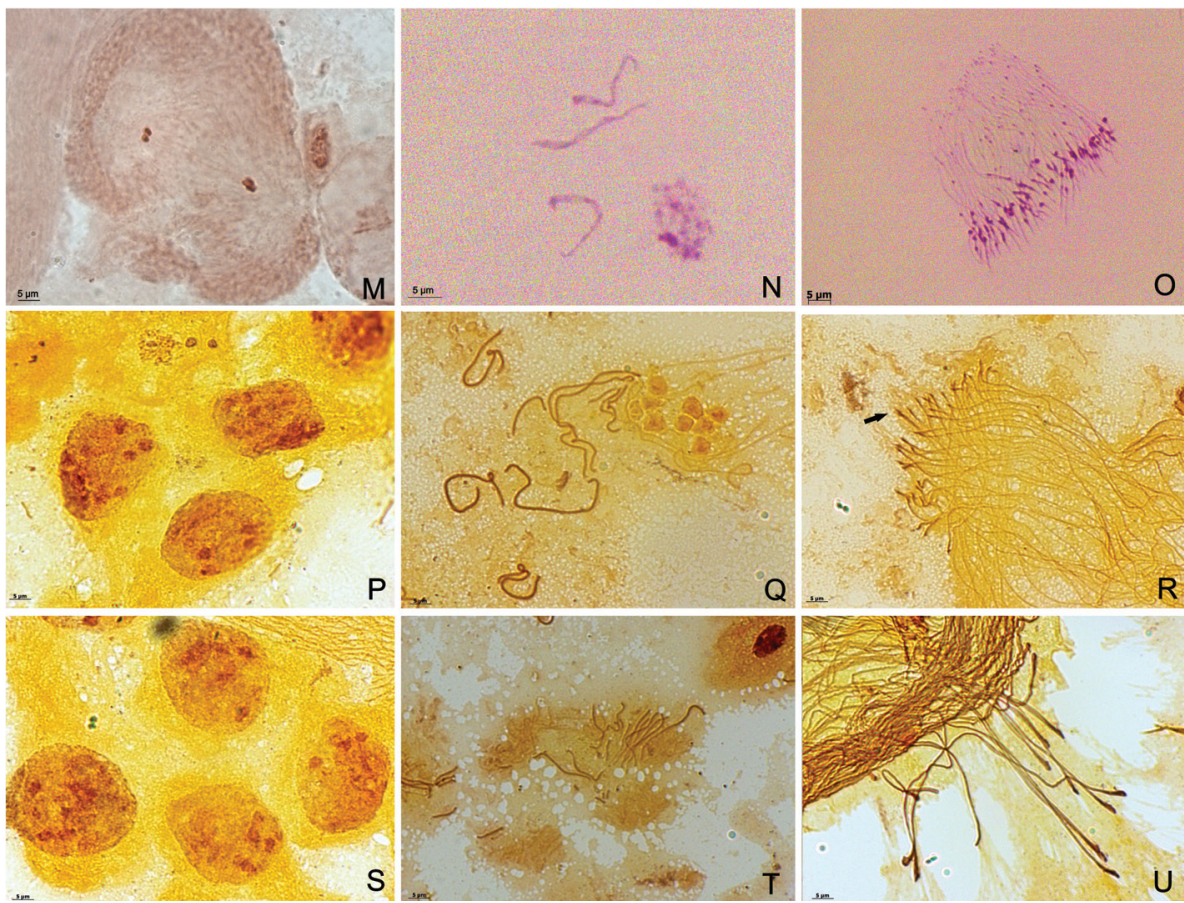

Figure 2 (cont.) - M. Telophase I showing the genetic material in the center of each nucleus. N. Elongated spermatids. O. Spermatozoid bundle presenting strong staining and a high concentration of chromosomal material at the tip of the bundles, this corresponding to the spermatozoid nuclei. (P-U). Testicular cells of Zaprionus indianus after silver impregnation. P. Spermatocytes in the initial state of prophase I, showing nucleolar bodies spread throughout the entire nucleus of each cell. Q. Spermatids in a more advanced stage of elongation not presenting specific impregnation along their entire length; R. Spermatozoids organized into long bundles with extremities strongly stained by AgNOR (arrow). (S-U) Testicular cells of Zaprionus sepsoides after silver impregnation. S. Spermatocytes in prophase I showing the nucleolar bodies of the cells revealed through silver impregnation; T. Spermatids in a more advanced stage of elongation not presenting specific impregnation along their entire length; U. Spermatozoids not showing strong AgNOR staining in one of their extremities. Scale bars: $5 \mu \mathrm{m}$.

tent number of 64 spermatozoids per bundle in both $Z$. indianus (Figures 4A-B) and Z. sepsoides (Figures 5A-B).

The ultrastructures of spermatozoids in both $Z$. indianus and $Z$. sepsoides revealed that the axonemes of both species possess a $9+9+2$ configuration, consisting of one pair of central microtubules, nine double peripheral microtubules formed by fibril A and fibril B, surrounded by nine additional accessory microtubules, plus nine spokes (Figures 4G-H and 5D-E, respectively). Additionally, proximal to the axoneme, two mitochondrial derivatives of different size were present (Figures 4B, 4D-G, and 5B-D) in both species. The larger mitochondrial derivative contained distinct paracrystalline material, particularly in $Z$. indianus (Figures 4D-E).

\section{Discussion}

The rapid dispersion of $Z$. indianus across different continents, and especially throughout Brazil, where this species is considered a pest on fig plantations (Stein et al.,
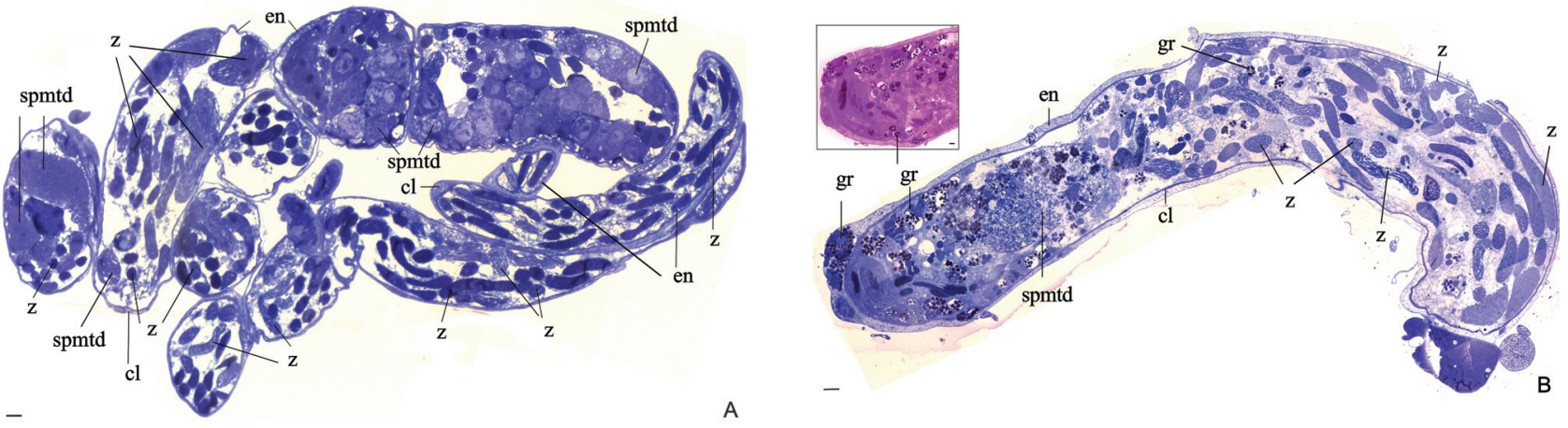

Figure 3 - Semi-thin histological sections of Zaprionus indianus (A) and Zaprionus sepsoides (B), showing spermatids (spmtd), followed by spermatozoid bundles arranged transversely and longitudinally (z). Other structures are also present in the testes, such as the coating layer (cl), the envelope (en) and glycogen granules (gr) revealed by P.A.S. reaction (note in detail of Figure B). Note these histological sections were $0.5 \mathrm{~mm}$ in length and stained with $1 \%$ toluidine blue. Scale bars: $10 \mu \mathrm{m}$. 

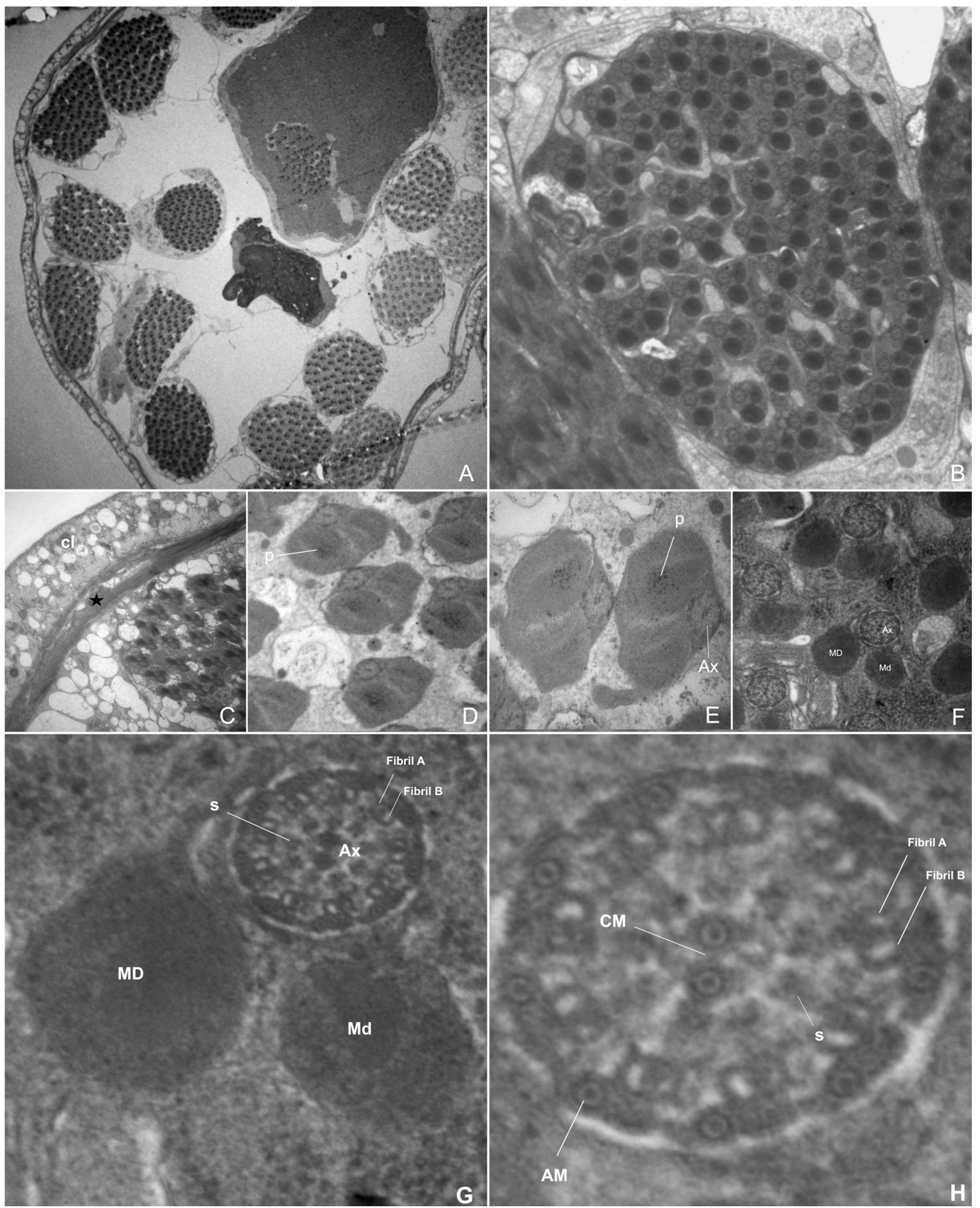

Figure 4 - TEM micrographs of spermatozoids (flagellum) of $Z$. indianus. A. Transverse section of the testes showing various spermatozoid bundles organized within cysts. B. Transverse section of the testes showing a bundle containing 64 spermatozoids in detail. C. Transverse section of the testes showing the coating layer (cl) and envelope (star). D-G. Transverse sections of a spermatozoid bundle, showing the tail region with axonemes (Ax) and mitochondrial derivatives of different size: larger mitochondrial derivatives (MD) in which the accumulation of paracrystalline material (p) is visible and smaller mitochondrial derivatives (Md). H. details of a flagellum showing the 9+9+2 arrangement: one pair of central microtubules (CM), nine double peripheral microtubules (formed by fibril A and fibril B), surrounded by nine additional accessory microtubules (AM) and nine spokes (s). Scale: Figure A: 2000x; Figure B: 10000x; Figure C: 8000x; Figures D, E, F: 67000x; Figure G: 84000x; Figure H: 100000x. 


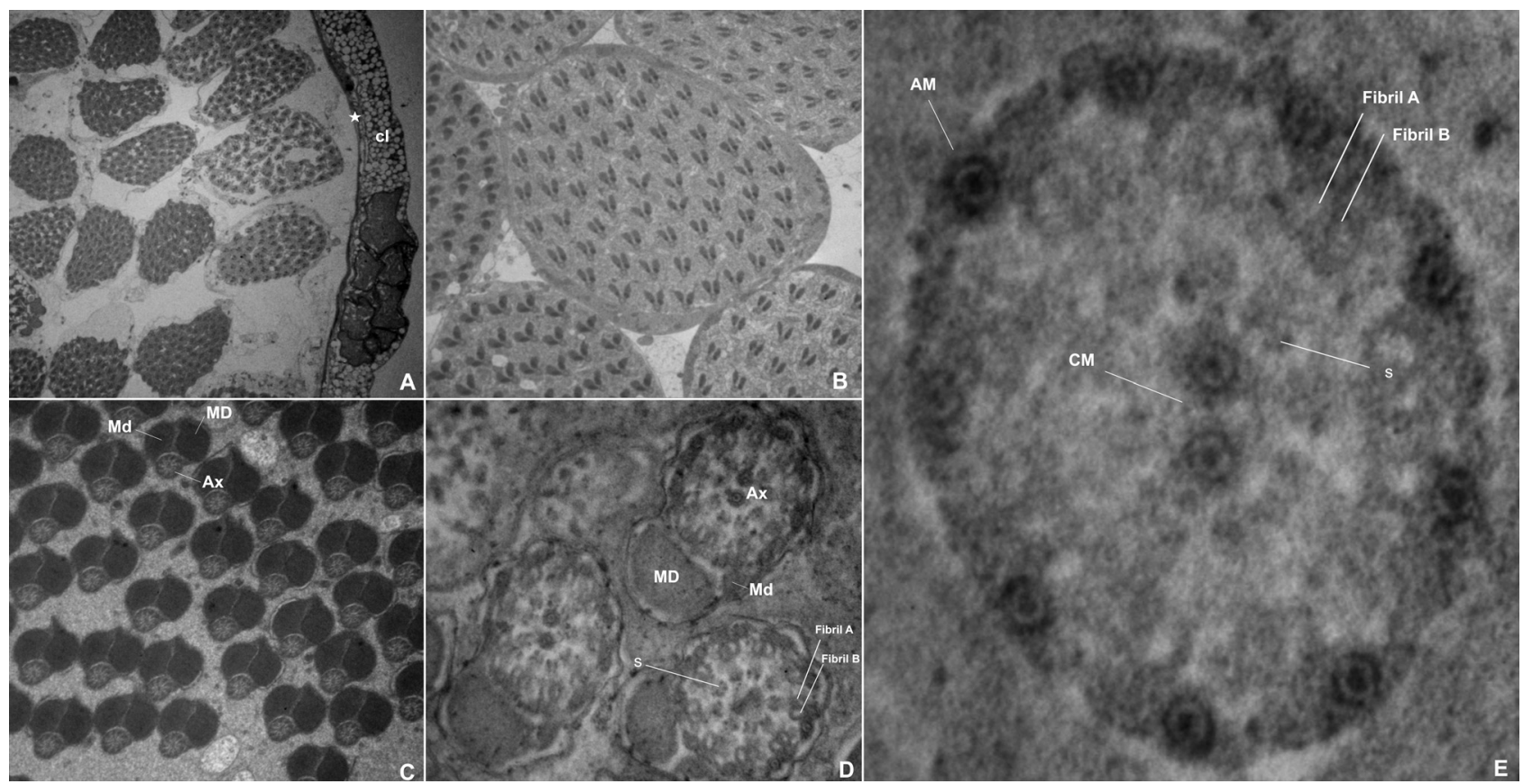

Figure 5 - TEM micrographs of spermatozoids (flagellum) of Z. sepsoides. A. Transverse section of the testes showing various spermatozoid bundles organized within cysts. B. Transverse section of the testes showing a bundle containing 64 spermatozoids in detail. C, D. Transverse section of a spermatozoid bundle, showing the tail region with axonemes $(\mathrm{Ax})$ and mitochondrial derivatives of different size: larger mitochondrial derivatives (MD) and smaller mitochondrial derivatives (Md) are visible, beside a coating layer (cl) and an envelope (star) E. details of a flagellum, showing the 9+9+2 arrangement: one pair of central microtubules (CM), nine double peripheral microtubules (note fibril A and fibril B), surrounded by nine additional accessory microtubules (AM) and nine spokes (s). Note the accumulation of electron-dense material in the nine accessory microtubules and in the two central microtubules. Scale: Figure A: 2000x; Figure B: 4000x; Figure C: 20000x; Figures D: 67000x; Figure E: 100000x.

2003), has spurred a growing number of studies on its biology and history of invasion. A similar reason motivated the present study on the spermatogenesis of this species in comparison to Z. sepsoides, as the two species differ in their ecological characteristics and testicular morphology. We showed that the testes of $Z$. indianus are formed by two coiled tubes of approximately $5 \mathrm{~mm}$ in length, whereas the testes of $Z$. sepsoides consist of two tubes of kidney shaped appearance of approximately $2 \mathrm{~mm}$ each. These measurements confirm those of Yassin and David (2010) obtained on testes of different species of Zaprionus. The inermis species can be classified into two categories: those with small testes, ranging from 1.0 to $2.0 \mathrm{~mm}$ in length (Z. sepsoides, Z. inermis, Z. cercus, Z. kolodkinae and Z. tsacasi), and those species with large testes, varying from 5.2 to $5.4 \mathrm{~mm}$ in length ( $Z$. indianus, $Z$. africanus and $Z$. taronus).

Araripe et al. (2004) showed that the anatomy and reproductive physiology of $Z$. indianus differs from $D$. melanogaster. For example, $Z$. indianus sperm are longer $(5 \mathrm{~mm})$ than the sperm of D. melanogaster $(1.8 \mathrm{~mm})$. Joly and Bressac (1994) measured the size of the sperm and testes of several drosophilid species and found that sperm size is approximately $6.7 \mathrm{~mm}$ in $Z$. indianus and $0.78 \mathrm{~mm}$ in $Z$. sepsoides and that the testicular sizes are $6.53 \mathrm{~mm}$ and 1.42 $\mathrm{mm}$, respectively. These measurements correspond to the testes measurements performed in this study.
The sperm bundles in Z. sepsoides were shorter than in $Z$. indianus, reflecting its smaller size and thus smaller testes. As frequently reported in the literature, testes are as long as the sperm they produce (Lindsley and Tokuyasu, 1980; Pitnick and Markow, 1994; Snook, 2005; Scharer et al., 2008).

The diploid number of $Z$. indianus and $Z$. sepsoides was confirmed to be $2 \mathrm{n}=12$ chromosomes. In this study, the AgNOR technique could not distinguish the nucleolar organizer regions (NORs) in the meiotic chromosomes. In prophase stage nuclei and in sperm, a NOR was intense protein synthesis in the anterior region, corresponding to the spermatozoid heads. Although this marking has been observed in studies of other organisms (Tavares and Azeredo-Oliveira, 1997; Tartarotti and Azeredo-Oliveira, 1999; Severi-Aguiar and Azeredo-Oliveira, 2005; Morielle-Souza and Azeredo-Oliveira, 2008; Costa et al., 2008; Peruquetti et al., 2008, 2010), few studies have examined AgNOR markings in the genus Zaprionus. Gupta and Kumar (1987) studied the association of polytene chromosomes with the nucleolus in four distinct Indian populations of Zaprionus indianus and observed that different chromosomes, such as $\mathrm{X}$ chromosome and microchromosomes, exhibit NOR activity.

In young $Z$. sepsoides males (1-3 days old), cells in the early stages of spermatogenesis, such as spermatocytes I and II, spermatids and a few spermatozoids, were ob- 
served more frequently than in young $Z$. indianus males. In contrast, the young $Z$. indianus males presented larger numbers of spermatids and spermatozoa and few cells at the initial stage of spermatogenesis. This finding suggests a difference in sperm maturation dynamics between the two species. In the genus Drosophila, spermatocytes enter in the first meiotic division in the pupal stage (Cooper, 1950), and the testes of most species of Drosophila are not fully developed at hatching and, with certain variability, continue to mature in the adults (Pitnick and Miller, 2000). The results of the present work indicate that a similar process occurs in Z. indianus, because one-day-old males already exhibited significant quantities of sperm bundles, whereas in $Z$. sepsoides such quantities are were only found in older males ( 8 days old).

These results contradict those of Pitnick and Miller (2000), who showed that in D. hydei, apparently selected for larger testes, there is an increase in development time (egg to adult) and time of maturation of spermatozoa posthatching, and this may contributes to a delay in reproduction of this population. In Z. indianus, which has larger testes and sperm than $Z$. sepsoides, this does not occur, because young males already present considerable amounts of mature sperm (Madi-Ravazzi, L.; David J., "personal communication")

The fertility of $Z$. indianus is greater than that of $Z$. sepsoides, at least in laboratory experiments (Madi-Ravazzi, L.; David J., "personal communication"), indicating a difference in the fitness of these species. If the size differences and maturation of sperm from these species favor greater reproductive potential, this phenomenon should be investigated in detail.

The organized distribution of spermatogenic cells observed in Z. indianus and Z. sepsoides is similar to that of other Drosophilidae (Cooper, 1950; Bairati, 1968; Meyer and Henning, 1974; Hardy et al., 1979; Lindsley and Tokuyasu, 1980; Fuller, 1993, Joly and Bressac, 1994; Gönczy and Dinardo, 1996, Li and Xie, 2005; Scharer et al., 2008; Mojica et al., 2000; Barreau et al., 2008, Cheng and Mruk, 2010).

Another relevant observation was the constant number of 64 sperm per bundle in both Z. indianus and $Z$. sepsoides. The mechanism underlying the formation of these bundles is similar to what occurs in Drosophila and other insects. In these species, a bundle of sperm is produced by a series of mitotic divisions of the spermatogonia within a spermatic cyst (Cooper, 1950; Hardy et al., 1979; Lindsley and Tokuyasu, 1980; Fuller, 1993; Fabrizio et al., 1998; Mojica et al., 2000).

Although the mitotic divisions within the cysts are synchronous in most insects, in some species of Drosophila the divisions can also be asynchronous and the number of sperm per bundle may vary from 32 , as in D. hydei, to 128 , as in D. pseudoobscura (Mojica et al., 2000). Similar ob- servations were made in bees (Cruz-Landim, 2001) and Coleoptera (Name et al., 2007).

According to Virkii (1969), basal orders of insects have more sperm per bundle than the more derived orders, and the more specialized groups tend to exhibit the lowest number of sperm per bundle. Name et al. (2007) demonstrated, for example, that certain scarab beetles have 128-512 sperm per bundle and Sitophilus zeamais and $S$. oryzae approximately 260 sperm per bundle. Fewer sperm per bundle indicates a reduced production of spermatozoa, which may correspond to a fitness that limits genetic variability (Mojica et al., 2000). Although $Z$. indianus and $Z$. sepsoides have the same number of sperm per bundle (64), there may be different numbers of cysts in these species, which is indicated by a greater abundance of sperm bundles in $Z$. indianus than in $Z$. sepsoides.

The present study confirmed the organizational pattern of the axoneme of insects following the 9+9+2 scheme, which is an arrangement of an internal 9+2 microtubules surrounded by nine additional accessory microtubule. Studies of the structural organization of the axoneme in Drosophila have been performed by various researchers (Perotti, 1969; Kiefer, 1970; Phillips, 1970; Dallai and Afzelius, 1991; Dallai et al., 1993; Fabrizio et al., 1998; Mojica et al., 2000), who all confirmed the conservation of this structure in different species of Drosophila and the family Drosophilidae. Two mitochondrial derivatives of different size are also present in both Zapronius species. In insects, these organelles have been studied ultrastructurally using a phylogenetic approach and this morphology of mitochondrial derivatives is observed in highy evolved species, being a apomorphic character (Phillips, 1970; Mojica et al., 2000). Some functions of mitochondrial derivatives may be related to the process of storing and releasing energy to power the mobility of the flagellum (Phillips, 1970, Lindsley and Tokuyasu, 1980).

Phylogenetic relationships of the genus Zaprionus has been extensively debated (Yassin et al., 2007, 2008, 2010, Yassin and David, 2010) and current reports agree that Zaprionus is more closely related to Drosophila than to the Sophopora subgenera, to which the melanogaster group belongs (Russo et al., 1995; Kwiatowski and Ayala, 1999; Da Lage et al., 2007; Commar et al., 2012). The ultrastructural morphology of the mitochondrial derivatives present in Zaprionus is very similar to those found in D. melanogaster. However, based on the above-cited studies, such similarity is possibly a homoplasy and not informative regarding the phylogenetic relationships of these taxa.

The findings presented here indicate differences in sperm maturation between the species analyzed. These differences may favor the reproductive success and fitness of $Z$. indianus and thus also relate to the invasive potential of the species. 


\section{Acknowledgments}

We thank Prof. Dr. Jean David (CNRS, Gif-SurYvette, France) for donating the $Z$. sepsoides strain, and Prof. Dr. Hermione E. Melara de C. Bicudo for valuable suggestions. Technical assistance for TEM was provided by José Augusto Maulim (Microscopy Center, Faculdade de Medicina de Ribeirão Preto, Universidade de São Paulo, Ribeirão Preto, SP). Financial support by the Coordenação de Aperfeiçoamento de Pessoal de Nível Superior (CAPES) is also acknowledged.

\section{References}

Amitin EG and Pitnick S (2007) Influence of development environment on male- and female- mediated sperm precedence in Drosophila melanogaster. J Compilation Eur Soc Evol Biol 20:381-391.

Amoudi MA, Diab FM and Abou-Fannah SSM (1991) Zaprionus indianus Gupta (Diptera, Drosophilidae) in Saudi Arabia and the effect of temperature on the life cycle. J King Saud Univ Agric 3:25-35.

Amoudi MA, Diab FM and Abou-Fannah SSM (1993a) Effects of larval population density on the life cycle parameters in Zaprionus indianus Gupta (Diptera, Drosophilidae). Pakistan J Zool 25:37-40.

Amoudi MA, Diab FM and Abou-Fannah SSM (1993b) The influence of low temperature on development, adult longevity and productivity of Zaprionus indianus Gupta (Diptera, Drosophilidae). J King Saud Univ Agric 5:263-274.

Araripe LO, Klaczko LB, Moreteau B and David JR (2004) Male sterility thresholds in a tropical cosmopolitan drosophilid, Zaprionus indianus. J Therm Biol 29:73-80.

Bairati A (1968) Structure and ultrastructure of male reproductive system in Drosophila melanogaster. II. Genital duct and accessory glands. Monit Zool Ital 2:105-182.

Barreau C, Benson E and White-Cooper H (2008) Comet and cup genes in Drosophila spermatogenesis: The first demonstration of post-meiotic transcription. Biochem Soc Trans 36:540-542.

Castro FL and Valente VLS (2001) Zaprionus indianus invading communities in the southern Brazilian city of Porto Alegre. Drosophila Inf Serv 84:15-17.

Chassagnard MT and Kraaijeveld AR (1991) The occurrence of Zaprionus sensu stricto in the Palearctic region (Diptera, Drosophilidae). Ann Soc Entomol Fr 27:495-496.

Cheng C and Mruk D (2010) The biology of spermatogenesis: The past, present and future. Phil Trans R Soc Lond B 365:1459-1463.

Commar LS, Galego LGC, Ceron, CR and Carareto CMA (2012) Taxonomic and evolutionary analysis of Zaprionus indianus and its colonization of Palearctic and Neotropical regions Genet Mol Biol 35:395-406.

Cooper KW (1950) Normal spermatogenesis in Drosophila. In: Demerec M (ed) The Biology of Drosophila. John Wiley and Sons, New York, pp 1-61.

Costa LC, Azeredo-Oliveira MTV and Tartarotti E (2008) Spermatogenesis and nucleolar Activity in Triatoma klugi (Triatomine, Heteroptera). Genet Mol Biol 31:438-444.
Cotta-Pereira G, Rodrigo FG and David-Ferreira JF (1976) The use of tannic acid-glutaraldehyde in the study of elastic related fibers. Stain Technol 51:7-11.

Cruz-Landim C (2001) Organization of the cysts in bee (Hymenoptera, Apidae) testis: Number of spermatozoa per cyst. Iheringia Sér Zool 91:183-189.

Da Lage JL, Kergoat GJ, Maczkowiak F, Silvain JF, Cariou ML and Lachaise D (2007) A phylogeny of Drosophilidae using the amyrel gene: Questioning the Drosophila melanogaster species group boundaries. J Zoolog Syst Evol Res 45:47-63.

Dallai R and Afzelius BA (1991) Sperm flagellum of Dacus oleae (Gmelin) (Tephritidae) and Drosophila melanogaster (Drosophilidae) (Diptera). Int J Insect Morphol Embryol 20:215-222.

Dallai R, Bellon PL, Lanzavecchia S and Afzelius BA (1993) The dipteran sperm tail: Ultrastructural characteristics and phylogenetics considerations. Zool Scr 22:193-202.

David JR, Araripe LO and Bitner-Mathé BC (2006) Quantitative trait analysis and geographic variability of natural populations of Zaprionus indianus, a recent invader of Brazil. Heredity 96:53-62.

de Setta N, Van Sluys MA, Capy P and Carareto CM (2009) Multiple invasions of Gypsy and Micropia retroelements in genus Zaprionus and melanogaster subgroup of the genus Drosophila. BMC Evol Biol 9:e279.

De Vaio ES, Cucci B, Castagnino AM, Franca ME and Martinez ME (1985) Meioitic differences between three triatominae species (Hemiptera, Reduvidae). Genetica 67:185-19.

Fabrizio JJ, Hime G, Lemmon SK and Bazinet C (1998) Genetic dissection of sperm individualization in Drosophila melanogaster. Development 125:1833-1843.

Fuller MT (1993) Spermatogenesis. In: Bate M and MartinezArias M (eds) The Development of Drosophila melanogaster. Vol. 1. Cold Spring Harbor Press, Cold Spring Harbor, pp 71-147.

Galego LGC and Carareto CMA (2007) Analysis of the drosophilid Zaprionus indianus introduction in Brazil: Contribution of esterase loci polymorphisms. Drosophila Inf Serv 90:79-84.

Galego LG and Carareto CMA (2010) Variation at the Est3 locus and adaptability to organophosphorous compounds in Zaprionus indianus populations. Entomol Exp App 13:97-105.

García-González F and Simmons LW (2007) Shorter sperm confer higher competitive fertilization success. Evolution 61:816-824.

Gönczy P and Dinardo S (1996) The germ line regulates somatic cyst cell proliferation and fate during Drosophila spermatogenesis. Development 122:2437-2447.

Goñi B, Fresia P, Calviño M, Ferreiro MJ, Valente VLS and Basso da Silva L (2001) First record of Zaprionus indianus Gupta, 1970 (Diptera, Drosophilidae) in southern localities of Uruguay, South America. Drosophila Inf Serv 84:61-65.

Gupta JP (1970) Description of a new species of Phorticella Zaprionus (Drosophilidae) from India. Proc Ind Nat Sci Acad 36:62-70.

Gupta JP and Kumar A (1987) Cytogenetics of Zaprionus indianus Gupta (Diptera, Drosophilidae): Nucleolar organizer regions, mitotic and polytene chromosomes and inversion polymorphism. Genetica 74:19-25. 
Hardy RW, Tokuyasu KT, Lindsley DL and Garavito M (1979) The germinal proliferation center in the testis of Drosophila melanogaster. J Ultrastruct Res 69:180-90.

Hosken DJ, Garner TWJ and Blanckenhorn WU (2003) Asymmetry, testis and sperm size in Yellow Dung Flies. Funct Ecol 17:231-236.

Howell WM and Black DA (1980) Controlled silver staining of nucleolus organizer regions with a protective colloidal developer: A 1-step method. Experientia 36:1014-1015.

Joly D and Bressac C (1994) Sperm length in Drosophilidae (Diptera): Estimation by testis and receptacle lengths. Int J Insect Morphol Embryol 23:85-92.

Kato CM, Foureaux LV, César RA and Torres MP (2004) Ocorrência de Zaprionus indianus Gupta, 1970 (Diptera, Drosophilidae) no estado de Minas Gerais. Ciênc Agrotec 28:454-455 (Abstract in English).

Kiefer BI (1970) Development, organization and degeneration of the Drosophila sperm flagellum. J Cell Sci 6:177-194.

Kwiatowski J and Ayala FJ (1999) Phylogeny of Drosophila and related genera: Conflict between molecular and anatomical analyses. Mol Phylogenet Evol 13:319 328.

Lachaise D and Tsacas L (1983) Breeding-sites in tropical African Drosophilids. In: Ashburner MHL Carson HL and Thompson JN (eds) The Genetics and Biology of Drosophila. Vol. 3a. Academic Press, London, pp 221-332.

Li L and Xie T (2005) Stem cell niche: Structure and function. Annu Rev Cell Dev Biol 21:605-31.

Lindsley DL and Tokuyasu KT (1980) Spermatogenesis. In: Ashburner MHL and Wright TRF (eds) The Genetics and Biology of Drosophila. Vol. 2b. Academic Press, New York, pp 225-287.

Mattos-Machado T, Solé-Cava AM, David JR and Bitner-Mathé BC (2005) Allozyme variability in an invasive drosophilid, Zaprionus indianus (Diptera, Drosophilidae): Comparison of a recently introduced Brazilian population with Old World populations. Ann Soc Entomol Fr 41:7-13.

Mello MLS and Vidal BC (1980) Práticas de Biologia Celular. FUNCAMP and Editora Edgard Blucher LTDA, Campinas, São Paulo, Brasil, 71 pp.

Meyer GF and Henning W (1974) The nucleolus in primary spermatocytes of Drosophila hydei. Chromosoma 46:121-144.

Mojica JM, File-Emperador S and Bruck DL (2000) Sperm bundle and spermatozoon ultrastructure in two species of the carding group of Drosophila. Invertebr Reprod Dev 37:147-155.

Morielle-Souza A and Azeredo-Oliveira MTV (2008) Study of the nucleolar cycle and ribosomal RNA distribution during meiosis in triatomines (Triatominae, Heteroptera). Micron 39:1020-1026.

Name KPO, Dos Reis GP and Baó SN (2007) An ultrastructural study of spermiogenesis in two species of Sitophilus (Coleoptera, Curculionidae). Biocell 31:229-236.

Okada T and Carson HL (1983) The genera Phorticella Duda and Zaprionus Coquillett (Diptera, Drosophilidae) of the Oriental region and New Guinea. Kontyu 51:539-553.

Perotti ME (1969) Ultrastructure of the mature sperm of Drosophila melanogaster Meig. J Submicro Cytol 1:171196.

Peruquetti RL, Assis IM, Taboga SR and Azeredo-Oliveira MTV (2008) Meiotic nucleolar cycle and chromatoid body forma- tion during the rat (Rattus norvegicus) and mouse (Mus musculus) spermiogenesis. Micron 39:419-425.

Peruquetti RL, Taboga SR and Azeredo-Oliveira MTV (2010) Characterization of Mongolian Gerbil chromatoid bodies and their correlation with nucleolar cycle during spermatogenesis. Reprod Domest Anim 45:399-406.

Phillips DM (1970) Insect sperm: Their structure an morphogenesis. J Cell Biol 44:243-277.

Pitnick S and Markow TA (1994) Large-male advantages associated with costs of sperm production in Drosophila hydei, a species with giant sperm. Evolution 91:9277-9281.

Pitnick S and Miller GT (2000) Correlated response in reproductive and life history traits to selection on testis length in Drosophila hydei. Heredity 84:416-426.

Russo CAM, Takezaki N and Nei M (1995) Molecular phylogeny and divergence times of drosophilid species. Mol Biol Evol 12:391-404.

Santos JF, Rieger TT, Campos SRC, Nascimento ACC, Félix PT, Silva SVO and Freitas FMR (2003) Colonization of northeast region of Brazil by the drosophilid flies Drosophila malerkotliana and Zaprionus indianus, a new potential insect pest for Brazilian fruitculture. Drosophila Inf Serv 86:92-95.

Scharer L, Dalage JL and Joly D (2008) Evolution of testicular arquiteture in Drosophilidae: A role for sperm length. BMC Evol Biol 8:e143.

Schmitz HJ, Valente VL and Hofmann PR (2007) Taxonomic survey of Drosophilidae (Diptera) from mangrove forests of Santa Catarina Island, southern Brazil. Neotrop Entomol 36:53-64.

Setta N and Carareto CMA (2005) Fitness components of a recently-established population of Zaprionus indianus (Diptera, Drosophilidae) in Brazil. Iheringia Sér Zool 95:47-51.

Severi-Aguiar GDD and Azeredo-Oliveira MTV (2005) Cytogenetic study on three species of the genus Triatoma (Heteroptera, Reduviidae) with emphasis on nucleolar organizer regions. Caryologia 58:293-299.

Snook RR (2005) Sperm in competition: Not playing by the numbers. Trends Ecol Evol 20:46-53.

Soto I, Corio C, Fanara JJ and Hasson E (2006) First record of Zaprionus indianus Gupta 1970 (Diptera, Drosophilidae) in Argentina. Drosophila Inf Serv 89:13-14.

Stein CP, Teixeira EP and Novo JPS (2003) Aspectos biológicos da mosca do figo, Zaprionus indianus Gupta, 1970 (Diptera, Drosophilidae). Entomotropica 18:219-221.

Tartarotti E and Azeredo-Oliveira MTV (1999) Pattern of nucleolar activity during spermiogenesis of two triatomines, Panstrongylus megistus and P. herreri. Caryologia 52:177184.

Tavares MG and Azeredo-Oliveira MTV (1997) Pattern of nucleolar activity during spermiogenesis in triatomines (Heteroptera, Reduviidae) as analysed by silver staining. Cytobios 89:93-103.

Tidon R, Leite DF and Leão BFD (2003) Impact of the colonization of Zaprionus indianus (Diptera, Drosophilidae) in different ecosystems of the Neotropical region: 2 years after the invasion. Biol Conserv 112:299-305.

Toni DC, Hofmann PRP and Valente VLS (2001) First register of Zaprionus indianus (Diptera, Drosophilidae) in the state of Santa Catarina, Brazil. Biotemas 14:71-85. 
Van der Linde K, Steck GJ, Hibbard K, Birdsley JS, Alonso L and Mand Houle D (2006) First records of Zaprionus indianus (Diptera, Drosophilidae), a pest species on commercial fruits from Panama and the United States of America. Fla Entomol 89:402-403.

Vilela CR (1999) Is Zaprionus indianus Gupta, 1970 (Diptera, Drosophilidae) currently colonizing the Neotropical region? Drosophila Inf Serv 82:37-39.

Virkii N (1969) Sperm bundles and phylogenies. Z Zellfosch 101:13-27.

Yassin A, Capy P, Madi-Ravazzi L, Ogereau D and David JR (2007) DNA barcode discovers two cryptic species and two geographical radiations in the invasive drosophilid Zaprionus (Diptera, Drosophilidae). Mol Ecol Resour 8:491-501.

Yassin A, Araripe LO, Capy P, Da Lage JL, Klaczko LB, Maisonhaute C, Ogereau D and David JR (2008) Grafting the mo- lecular phylogenetic tree with morphological branches to reconstruct the evolutionary history of the genus Zaprionus (Diptera, Drosophilidae). Mol Phylogenet Evol 47:903915.

Yassin A, Da Lage JL, David JR, Kondo M, Madi-Ravazzi L, Prigent SR and Toda MJ (2010) Polyphyly of the Zaprionus genus group (Diptera, Drosophilidae). Mol Ecol Resour 55:335-339.

Yassin A and David JR (2010) Revision of the Afrotropical species of Zaprionus (Diptera, Drosophilidae), with descriptions of two new species and notes on internal reproductive structures and immature stages. ZooKeys 51:33-72.

Associate Editor: Carlos R. Machado

License information: This is an open-access article distributed under the terms of the Creative Commons Attribution License, which permits unrestricted use, distribution, and reproduction in any medium, provided the original work is properly cited. 